\title{
ELECCIÓN DE SALA CUNA: ¿CUÁLES SON LOS REQUERIMIENTOS DE LOS PADRES?
}

\author{
Choosing daycare: what are parents's choices? \\ Escolha de berçário: quais são as exigências dos pais? \\ Claudia Oriana Silva \\ Universidad de Playa Ancha, Chile. Correo electrónico: silva.claudia.2007@gmail.com
}

\section{Resumen}

La inserción de la mujer al mercado laboral ha causado que el cuidado de niños/as menores de dos años en nuestro país crezca. Y no sólo el cuidado, sino que la estimulación y los aprendizajes, es decir, que las Salas Cuna aporten en el desarrollo integral de los niños/as. Es por esto que se decidió investigar cuáles son los requerimientos que tienen los padres al momento de elegir una Sala Cuna adecuada para sus hijos.

Los resultados de la investigación presentaron que de la muestra, el 85\% hace uso de este servicio por su trabajo y en un 55\% el ingreso a este nivel educativo es después del año de edad. Según la opinión de los padres, lo más relevante de estas instituciones son los cuidados a su hijo/a, seguido por otorgar tranquilidad mientras trabajan.

En relación a lo que los padres buscan de una Sala Cuna, se obtiene como prioridad la seguridad y como recurso adicional, un sistema de cámaras de vigilancia para padres. En cuanto a la percepción de lo que debe entregar una Sala Cuna ideal, los padres priorizan en entregar aprendizajes de calidad y la importancia de cuidados hacia los niños y niñas.

Palabras clave: sala cuna, infancia, mujer trabajadora.

\begin{abstract}
The insertion of women in the labor market has caused the increase of children's care in our country. And not only the care, but the stimulation and learning, that is, the Nursery Schools contribute to the overall development of children. That's why I decided to investigate what are the requirements that parents have when they have to choose a suitable nursery for their children. The results of the research showed that $85 \%$ of the sample makes use of this service because of their work and $55 \%$ of the entry at this level of education is after one year old.
\end{abstract}


According to the parents's opinion, the most relevant aspect of these institutions are the quality of care given to their children, followed by the tranquility they have while they stay in their workplaces. In relation to what parents are looking for in a Daycare, security is priority, and as an additional resource, a system of surveillance cameras for parents. Regarding the perception of what an ideal Nursery should give to the children, parents prioritize in the quality of learnings and the importance of children's care.

Keywords: daycare, childhood, worker woman.

\section{Resumo}

A inserção das mulheres no mercado de trabalho fez que o atendimento de crianças pequenas em nosso país crescesse. E não apenas o cuidado, mas o estímulo e a aprendizagem, ou seja, os Berçarios contribuem para o desenvolvimento global das crianças. É por isso que eu decidi investigar quais são os requisitos que os pais têm quando escolhem um lugar adequado para seus filhos.

Os resultados da pesquisa mostraram que $85 \%$ da mostra faz uso desse serviço a causa do seu trabalho e 55\% da renda nesse nível de ensino é após um ano de idade. Na opinião dos pais, a mais relevante dessas instituições é o cuidado de seus filhos, seguido de tranquilidade enquanto trabalham.

Em relação ao que os pais estão procurando em um Berçario, a prioridade é dada à segurança e, como recurso adicional, um sistema de câmeras de vigilância para os pais. Em relação à percepção do que um berçário ideal deve oferecer, os pais priorizam o aprendizado de qualidade e a importância do cuidado com a criança.

Palavras-chave: berçário, infância, mulher trabalhadora.

\section{Identificación del problema}

Hoy en día, el acceso a las Salas Cunas es considerado una necesidad, la realidad actual se caracteriza por el cambio constante e incesante por la gran cantidad de información que se maneja, como la globalización y el consumismo. Nos encontramos entonces con una sociedad en la que tanto hombres como mujeres están orientando muchos de sus esfuerzos en competir, en perfeccionarse a través del estudio y trabajo, siendo muchos los padres y/o madres los que deben salir a trabajar, haciéndose indispensable el servicio que ofrecen las 
Salas Cunas. "La inmersión de la mujer al mundo laboral, ya sea para contribuir en el sustento del hogar o bien, para realizarse profesionalmente, dedicando varias horas del día a su trabajo. Razones por las cuales la Educación Parvularia adquiere una gran relevancia. Considerando que la temprana infancia es una etapa del desarrollo humano, que permite instaurar las bases de los futuros aprendizajes que se irán adquiriendo en las diversas dimensiones de la vida.” (Cortes, C. Navarrete, E. Troncoso, M, 2009:7)

Es así como la modernidad y la incorporación de la mujer al mundo del trabajo favorece la asistencia de los niños/as mientras sus madres trabajan, especialmente en familias monoparentales de bajos ingresos. "En Chile a partir del reciente aumento en la cobertura para la atención de niños/as menores de tres años en las Salas Cunas y jardines infantiles es una tendencia creciente. La asistencia de los párvulos busca igualar oportunidades de desarrollo, especialmente de aquellos niños/as más vulnerables” (Olhaberry, 2011: 166)

Las circunstancias que se viven con frecuencia en la sociedad actual a nivel nacional nos obligan a examinar la educación infantil, teniendo en cuenta que el quehacer pedagógico siempre supone una intención. Por su parte; la convención de los derechos del niño consagra en su artículo 28 el derecho a la educación.

En ese sentido, el rol de la Educación Parvularia es medular, siendo tal el impacto de la educación en la primera infancia que la búsqueda constante de los padres por encontrar un lugar que ofrezca un servicio de calidad se hace indispensable. Un aspecto relevante al evaluar el efecto de los cuidados alternativos es la calidad de la atención que reciben, considerando diversos componentes relevantes para entregar programas de Educación Parvularia de calidad. "Entre estos factores, destacan los relativos a la estructura (infraestructura, proporción de adultos por niños/as, adultos capacitados, ambientes físicos seguros, tamaño de la sala, materiales disponibles, etc.) y aquellos referentes a procesos (interacciones en sala, ambientes lingüísticos enriquecidos, currículos apropiados, etc.)” (Rolla \& Rivadeneira, 2006; Bedregal, 2006; Banco Mundial, 2009; Bennet, 2008; Center on the Developing Child, 2005, cit por Casas, L. Valenzuela, E, 2012: 142)

La evidencia es clara en definir los principales aspectos que se relacionan con la calidad de la Educación Parvularia, considerando por un lado la calidad estructural; es decir coeficiente técnico y ambiente físico. Y calidad de los procesos como lo es la interacción 
entre niño/a y el adulto y la metodología de trabajo. El énfasis radica en la necesidad de atender estos factores a la hora de desarrollar programas, planes y políticas relacionadas con niños/as en edad temprana.

En Chile, para las mujeres que trabajan, los derechos de Sala Cuna, alimentación y cuidado del hijo menor son irrenunciables. Las necesidades de la sociedad actual obligan tanto al hombre como a la mujer a crecer en lo personal como en lo profesional, introduciéndose en actividades que demandan una cantidad de tiempo extra, lo que implica menor tiempo de entrega para el cuidado y educación de los hijos/as, siendo muchos los padres y/o madres quienes deben salir a trabajar por lo que se hace indispensable el servicio que ofrecen las Salas Cuna.

Una de las funciones de estas es que permiten que los padres compatibilicen el desarrollo de su trabajo con la atención de sus hijos/as, cumpliendo un rol muy importante de apoyo para las madres y/o padres que deben salir a trabajar, pasando a ser un agente educativo importante en la etapa inicial de los hijos/as.

Los niños/as de nuestro país, desde los 3 meses hasta los 2 años son atendidos en Salas Cunas de instituciones como la Fundación Integra, la Junta Nacional de Jardines Infantiles (JUNJI), Vía Transferencia JUNJI (VTF) y Particulares. Además de la escasa oferta para el número de demanda existente se suma a esto que no responden a los requerimientos de los padres en cuanto a calidad y sistemas modernos, que permitirían entregar una mejor educación a los lactantes como además una confianza a sus progenitores.

Según un estudio del año 2016 realizado por el Centro de Investigación Avanzada en Educación (CIAE) de la Universidad de Chile y el Centro de Inteligencia Territorial (CIT) de la Universidad Adolfo Ibáñez, en la ciudad de Santiago faltan 33.653 vacantes para salas cuna (niños de 0 a 2 años), y se necesitan otros 45.688 cupos para los niveles medio menor y medio mayor (de 2 a 4 años).

Los resultados de la Encuesta Casen del 2013 arrojan que aproximadamente un 89\% de los niños menores de 2 años no asisten a sala cuna.

"Las encuestas muestran que las principales razones por las que los/as niños/as no asisten a salas cuna y jardines infantiles son la preferencia por el uso de otro tipo de arreglos 
de cuidado; fuertes convicciones de los hogares de que los centros no son buenos para los/as niños/as; calidad y confianza en el servicio ofrecido; falta de información sobre los beneficios potenciales en términos de estimulación temprana; precio y disponibilidad de los centros; y horarios y distancia de la casa o el trabajo.” (Mercedes, M. Díaz, M. Vásquez, J, 2016:14)

Es por esto que se hace necesario ofrecer un servicio integro de cuidado para los párvulos, con jornadas de extensión, con un sistema de información constante, con modernos $\mathrm{y}$ atractivos sistemas de seguridad, con infraestructura y personal idóneo para fortalecer la confianza de los padres con el fin de entregar un servicio único con educación de calidad.

El derecho a la Sala Cuna es un beneficio de gran ayuda a las madres trabajadoras. Sin embargo, los derechos que se conocen hoy en día, se fueron adquiriendo de manera paulatina. El surgimiento de la protección a las trabajadoras en la legislación chilena, nace en el contexto del reconocimiento de la lucha creciente del proletariado por mejores condiciones y una vida digna en el proceso de industrialización del país, en donde la tasa de mortalidad de la primera infancia tenía un gran porcentaje, por lo que el postnatal, derecho a Sala Cuna y otras normas de protección son reconocidas.

El modelo de industrialización basado en el hombre proveedor no repara en que exista un alto porcentaje de mujeres realizando labores remuneradas. Ya en la última década comienza a aparecer una creciente preocupación por la protección de la familia, los roles de crianza, el cuidado infantil, su relación con el equilibrio entre parentalidad, trabajo y apego. Estos cambios culturales surgen con la inserción de la mujer al mundo productivo, a la política, cargos públicos y diversos trabajos remunerados como manifestación de su igualdad en los diversos ámbitos de la vida pública y privada.

"La tasa de ocupación femenina es de 45,8 mientras que la masculina 72,4. El aumento al inicio de los noventa es claro, pero no se puede dejar de considerar la segregación sexual del mercado, las mujeres se concentran en comercio, servicio doméstico y educación” (Casas, B. Lidia. Valenzuela, Ester, 2012:3)

Mientras la mujer se insertaba en el mercado laboral el cuidado, atención y estimulación de los niños no estaba a cargo de personal idóneo o en condiciones que realmente fueran las adecuadas. 
Pieper critica la consagración de los derechos de Sala Cuna y lactancia, puesto que no se consideró el contexto de las trabajadoras, debido a las dificultades de trasladar a sus hijos/as o el estigma social asociado a dejar a los hijos "abandonados" en las Salas Cuna que no cumplían con las necesidades de las madres trabajadoras, por lo que surgía la necesidad de buscar medios alternativos para el cuidado infantil.

Es así como la primera infancia toma creciente relevancia a nivel nacional e internacional, numerosas investigaciones en psicología, educación, neurociencias y economía han resaltado los beneficios de una adecuada estimulación temprana en el desarrollo y aprendizaje de los niños/as.

\section{La investigación}

La presente investigación se sustenta en un enfoque cuantitativo. En este sentido, la investigación permitió generalizar datos y luego cuantificarlos, en relación a los requerimientos actuales que tienen los padres al momento de elegir una Sala Cuna, temática que ha sido poco conocida y difundida desde el ámbito pedagógico.

Se procedió a recopilar información sobre los requerimientos que manifiestan los padres ante la elección de una Sala Cuna aplicando un cuestionario a la muestra seleccionada. En ambas instancias se evidencia el tipo de investigación exploratoria, que abrirá las puertas a la modalidad descriptiva.

Responde al tipo de estudio Descriptivo, ya que se realizó un análisis de los requerimientos de los padres al elegir una Sala Cuna, buscando interpretar los resultados para dar cuenta de la realidad actual de los establecimientos seleccionados.

Ante los datos entregados anteriormente, con este estudio se pretendía dilucidar lo siguiente:

¿Cuáles son los requerimientos actuales de los padres al momento de elegir una Sala Cuna Particular para sus hijos?

La presente investigación se realizó con un muestreo no probabilístico del tipo por conveniencia ya que permite un fácil acceso a estas instituciones y además se interesan por cooperar en la presente investigación. 
El criterio de selección son Salas Cuna Particulares ubicadas en el sector de Llolleo, comuna de San Antonio que se encuentren bajo el reconocimiento de normativa JUNJI. Siendo esta normativa un indicador de calidad; ya que supervisa el correcto funcionamiento de una Sala Cuna.

Las que cumplen con este criterio son:

\begin{tabular}{|l|c|l|c|}
\hline \multicolumn{1}{|c|}{ Nombre Sala Cuna } & Rol JUNJI & Dirección & $\begin{array}{l}\text { Número apoderados Nivel } \\
\text { Sala Cuna }\end{array}$ \\
\hline $\begin{array}{l}\text { Sala Cuna y Jardín Infantil } \\
\text { Castillito de Sonrisas. }\end{array}$ & 235 & Santa Lucia 520 Llolleo. & 14 \\
\hline $\begin{array}{l}\text { Sala Cuna y Jardín Infantil } \\
\text { My First Step Garden. }\end{array}$ & 1568 & Santa Rita 1411 Llolleo. & 6 \\
\hline
\end{tabular}

Tabla $\mathrm{N}^{\circ}$ 1. Elaboración propia.

Es necesario considerar los cambios culturales y sociales que se han generado en nuestro país, en donde la inserción de la mujer al mercado laboral y la política pública en educación fomentan la inserción de párvulos menores de dos años al nivel de Sala Cuna. Este nivel educativo ha tomado relevancia a través de investigaciones respecto al desarrollo neuronal del niño/a en sus primeros años de vida, posibilitando además la igualdad de oportunidades en la primera infancia a través de ampliación de cobertura y asistencia a nuevas instituciones.

\subsection{El instrumento}

Se elaboró y aplicó una encuesta de 21 preguntas cerradas. Estas preguntas presentan alternativas de respuestas delimitadas. Como además opciones donde se debe asignar puntaje de 1 al 5 siendo 1 muy importante y 5 muy poco importante.

El instrumento aplicado fue el siguiente:

En las siguientes preguntas de la 1 a la 13. Marque con una cruz solo 1 alternativa. Recuerde, NO se deben
marcar más opciones.
1. ¿Qué relación tiene usted con el párvulo de Sala Cuna?
a) Madre
b) Padre
c) Otro familiar.
d) Apoderado.
¿Cuál es rango de edad del Padre o su figura paterna?
a) Padre entre 15 a 25
b) Padre entre 26 a 36
c) Padre entre 37 a 47
d) Más edad.


3. ¿Cuál es rango de edad de la Madre o su figura materna?
e) Padre entre 15 a 25
f) Padre entre 26 a 36
g) Padre entre 37 a 47
h) Más edad.

4. ¿Cuál es el nivel educacional del Padre o figura paterna?
a) Enseñanza básica completa
b) Enseñanza básica incompleta
c) Enseñanza media completa.
d) Enseñanza media incompleta
e) Técnico profesional completa.
f) Técnico profesional incompleto
g) Universitaria completa.
h) Universitaria incompleta
i) Postgrado.

5. ¿Cuál es el nivel educacional de la Madre o figura materna?
a) Enseñanza básica completa.
b) Enseñanza básica incompleta
c) Enseñanza media completa.
d) Enseñanza media incompleta
e) Técnico profesional completa.
f) Técnico profesional incompleto
g) Universitaria completa.
h) Universitaria incompleta
i) Postgrado.

6) Él párvulos que asiste a Sala Cuna ¿Cuántos hermanos/as tiene?
a) No tiene hermanos/as.
b) 1
c) 2
d) 3 o más.

7) El párvulo que asiste a Sala Cuna ¿Qué ubicación ocupa entre sus hermanos?
a) Primero
b) Segundo
c) Tercero
d) Cuarto o más

8) ¿Por qué su hijo/a asiste a la Sala Cuna?
a) Por trabajo.
b) Para tener un tiempo personal.
c) Para que me orienten en como estimular a mi hijo/a.
d) Para que logre socializar y compartir con otros niños/as

9) Para llegar a la Sala Cuna usted necesita:
a) Caminar algunas cuadras.
b) Movilizarse en un vehículo unos 15 minutos.
c) Movilizarse en un vehículo más de 15 minutos.
d) Desplazarse de una comuna a otra.

10) ¿A qué edad inscribiría o inscribió a su hijo/a en una Sala Cuna?
a) 84 días.
b) 6 meses.
c) Entre 1 año y 1 año 6 meses.
d) Mayor a un 1 año 6 meses.

11) ¿Qué jornada prefiere para la asistencia a la Sala Cuna?
a) Mañana
b) Tarde
c) Jornada completa
d) Extensión horaria.

12) Para Usted ¿Cuál es una cantidad adecuada de niños/as por sala? 

a) $21-25$
b) $15-20$.
c) $10-14$.
d) $9-1$

13) Según su opinión ¿Cuál es una cantidad adecuada de adultos; considerando la educadora y técnicos por niño/a?

a) 1 adulto por cada 6 niños/as.

b) 1 adulto por cada 5 niños/as.

c) 1 adulto por cada 3 niños/as.

d) 1 adulto por cada 2 niños/as

En las siguientes preguntas priorice las opciones siendo 1 lo más importante y 5 lo menos.

14) ¿Qué considera más importante de una Sala Cuna?

Entregar cuidados a mi hijo/a.

Brindar aprendizajes a mi hijo/a.

Otorgar tranquilidad mientras trabajo.

Ser apoyo complementario a la familia en la educación.

Realizar actividades extraprogramáticas con la familia.

15) Según su percepción, una Sala Cuna ideal debe entregar a los párvulos.

Aprendizajes de calidad.

Cuidados básicos de higiene y alimentación.

Entretención a los párvulos.

Independencia a los párvulos.

Interacción con otros niños/as.

16) ¿Qué considera necesario para el correcto funcionamiento de una Sala Cuna?

Personal adecuado.

Orden y limpieza.

Proyecto educativo.

Realización de actividades a los párvulos.

Tener reconocimiento JUNJI.

17) Según usted. Es importante que su Sala Cuna realice actividades con los niños/as siendo más importante qué:

Sean de estimulación sensorial, motriz y del lenguaje.

Sean basadas en el juego.

Sean de cuidados.

Tengan materiales concretos y llamativos.

Propicien la convivencia con otros niños.

18) ¿Qué busca de una Sala Cuna para su hijo/a?

Seguridad.

Infraestructura moderna.

Mobiliario adecuado.

Materiales llamativos.

Personal Capacitado que realice actividades diversas.

19) ¿Con qué recursos le gustaría que contara una Sala Cuna?

Cámaras de vigilancia para padres.

Vías de evacuaciones modernas y seguras.

Sitio web con certificados e informes de avance en línea.

Blog con recomendaciones de trabajo en el hogar.

Implementos llamativos y seguros para los niños/as.

20) ¿Qué condiciones considera a la hora de elegir una Sala Cuna para sus hijos/as?

Ubicación

Seguridad

Precio

Infraestructura

Recomendación por otros padres.

21) De las siguientes actividades cual le interesa a ud que le brinde la Sala Cuna.

Talleres para padres con diferentes profesionales. 
Recomendaciones de estimulación enviadas al hogar. Citaciones personales para resolver dudas. Actividades extraprogramaticas. Actividades en sala con apoderados y niños/as.

\section{Resultados}

A continuación, se darán conocer los principales hallazgos que dan cuentan del logro de los objetivos planteados respecto a las decisiones de los padres de niños/as menores de dos años al momento de seleccionar una Sala Cuna.

Las motivaciones de los padres al momento de elegir una Sala Cuna corresponden:

- A su necesidad de conciliar vida laboral y crianza, lo cual corresponde también a un derecho, que se refleja con la ley 20.166 que consagra e independiza los derechos de Sala Cuna y alimentación e introduce flexibilidad en los tiempos de amamantamiento. Esta ley alude a la protección de la vida y la salud del menor, sin desmedro de la tranquilidad física y mental de la madre mientras trabaja. Beneficio social en nuestro país, que establece que el derecho a Sala Cuna es hasta que el menor cumpla los dos años de edad, para todo establecimiento que cuente con 20 o más mujeres trabajando.

- En la preferencia por la jornada completa, esto tiene directa relación con su horario de trabajo. En la actualidad las Salas Cunas logran satisfacer estas necesidades de los padres y empresas, al ofrecer sistemas de jornadas completas, parciales, extensiones y flexibilidad dentro de la semana o incluso fines de semana, adecuándose a las diferentes necesidades durante todo el año, sin excepción de vacaciones.

- El Preferir matricular a sus hijos/as cuando tienen más de 1 año de edad, siendo respaldado con estudios que mencionan que los padres de los niños/as más pequeños tienen una mayor tendencia a usar estrategias de cuidado informales, cuidado de familiares o madres que se dedican a cuidar a su hijo/a, para no dejarlos en un centro educativo tan pequeños. Esto a la vez provoca una menor tasa de ocupación de las madres entre menos edad tenga el niño/a.

- Que exista Menor cantidad de niños/as por aula, requiriendo cursos de 10 a 14 niños por sala. Esto no coincide con la normativa JUNJI, ya que la capacidad de atención 
es hasta 20 párvulos, siendo este el máximo que optan las Salas Cunas particulares al acreditarse y que tengan el espacio físico indicado según la normativa vigente que es de 2,5 metros cuadrados por niño/a.

- Una Atención personalizada, considerando que es adecuado 1 adulto por cada 3 niños/as; por su parte, la normativa vigente en el Decreto Supremo $N^{\circ} 315$, última modificación D.S. $\mathrm{N}^{\circ} 115$, MINEDUC, que regula las condiciones en la Educación Parvularia, menciona que el personal mínimo requerido es una educadora de párvulos por hasta 42 lactantes divididos en máximo dos grupos y una técnica en párvulos cada 7 menores. Los centros educativos donde se realizó el estudio trabajan con una educadora de párvulos en aula para 20 lactantes y un técnico en párvulos por cada 7 menores, entregando una atención más personalizada que otras instituciones educativas.

En cuanto a las prioridades de los padres al momento de elegir una Sala Cuna se refieren a:

- Cuidados a su hijo/a y otorgar tranquilidad mientras trabajan; De aquí la importancia del acceso a Sala Cuna y la atención integral que entregan a los párvulos, desde la atención de necesidades básicas de higiene, alimentación y sueño, como las de aprendizajes. Los hijos/as tienen un efecto considerable, este aspecto es vital en la decisión laboral de la madre de armonizar trabajo y maternidad lleva a muchas mujeres a que decidan no trabajar. En los últimos años estos centros educativos han permitido incrementar el nivel de empleo femenino y la incorporación de la mujer al mundo laboral, compatibilizando el desarrollo de su trabajo con el cuidado de sus hijos.

- La Seguridad; en relación a esto se encuentra el Manual de seguridad y prevención de riesgos de JUNJI y MINEDUC, la cual entrega directrices para que cada institución elabore sus planes y protocolos, entendiéndose la prevención de riesgos y seguridad como un tema que se vincula al funcionamiento global de las unidades educativas, siendo una implicancia directa en su gestión técnica administrativa. Como objetivo transversal la seguridad y prevención de riesgos de accidentes en los párvulos es un tema que debe estar presente en todo el currículum y debe 
comprometer a toda la comunidad educativa. Por su parte la dirección de obras municipales tiene sus exigencias antes de otorgar una recepción final, donde se considera el tipo de edificación, orden sanitario, emplazamiento según las normas del plan regulador, siendo esta entidad la que autoriza y aprueba el funcionamiento de las edificaciones para el destino determinado. Además de las exigencias de contar con vías de evacuación según decreto supremo $\mathrm{N}^{\circ} 594$ MINSAL y vías de escapes como puertas amplias de apertura hacia afuera, indicadas en decreto supremo $\mathrm{N}^{\circ} 289$ MINSAL. Junto a esto se certifica que el establecimiento cumple con las medidas de seguridad por medio de la visita y certificado emitidos por el cuerpo de bomberos.

- Que se desarrollen Aprendizajes de calidad y experiencias educativas de estimulación sensorial, motriz y lenguaje, es necesario considerar como aporte importante las investigaciones desde las neurociencias, las que demuestran que los efectos del ambiente son significativos y condicionantes en el proceso del llamado neurodesarrollo, la razón de ello recae en el ambiente y tiene gran impacto en los primeros momentos de la vida, en donde las experiencias tempranas interactúan en forma permanente y dinámica en el desarrollo cerebral. A los dos años el cerebro del niño/a tiene el doble de conexiones sinápticas que un adulto, por lo que es necesario tener ciertos tipos de estimulación para desarrollarse propiamente incluyendo áreas como el lenguaje, pensamiento y visión, ante lo cual su permanecía en una Sala Cuna tiene gran impacto a nivel neuronal mientras más significativas sean las experiencias en aula mayores serán las conexiones sinápticas del menor.

"La evidencia científica proveniente de la Neurociencia, ha individualizado los años preescolares como críticos para desarrollar acciones que influirán en forma decisiva en el desarrollo posterior del individuo, tanto en sus capacidades físicas y mentales como en su personalidad y desarrollo social.” (Cortes, C. Navarrete, E. Troncoso, M, 2009:37)

El trabajo realizado en las Salas Cuna es de facilitar diversas experiencias planificadas y organizadas con intencionalidad pedagógica para el niño/a explore, asocie, compare, experimente y juegue acrecentando todas sus potencialidades. Todas estas experiencias entregadas favorecen su desarrollo integral, desde su conocimiento corporal, emociones, su entorno social y familiar, interacciones y la sociabilización entre otros aspectos relevantes 
para su desarrollo y en estos centros educativos se propician en forma organizada por medio de diversas actividades.

- La existencia de un sistema de cámaras para padres; este aspecto se encuentra prohibido por la Superintendencia de Educación en el dictamen $N^{\circ} 6$ del 23 de Diciembre 2014, en donde la grabación de actividades en aula están autorizadas exclusivamente para efectos de la evaluación docente, en el sector particular como lo son las instituciones de la muestra, la inspección del trabajo refiere que la utilización como vigilancia o fiscalización de la actividad del trabajador no resulta lícita, constituyendo una intromisión no idónea y desproporcionada de la esfera íntima, haciendo inexistente la libertad como derechos de la constitución. Las directrices del Ministerio de Educación de nuestro país promueven un ambiente tolerante y de respeto hacia la integridad física, psíquica y moral de los educandos. En este sentido, la prioridad de los establecimientos educacionales debe favorecer la calidad por medio de planificación y su proyecto educativo institucional.

\section{Proyecciones}

La compatibilización de maternidad y trabajo es uno de los principales problemas a los que se ven enfrentadas las madres. En Chile para facilitar esa conciliación de roles se ha priorizado el acceso a Sala Cuna, como beneficio de las madres trabajadoras, por sobre un mejoramiento de la política de permisos posnatales.

La política en cuestión debería apostar por un posnatal más largo, ya que según los análisis de los cuestionarios, la mayoría de las madres considera que a la edad de 1 año es adecuado el ingreso a la Sala Cuna y desde un punto de vista teórico, un aumento del posnatal debería aumentar la oferta laboral femenina, ya que las mujeres se verían enfrentadas a una regulación más amigable con sus necesidades particulares, sin la necesidad de hacer uso de licencias por enfermedad del niño menor de un año. Además, podría visualizarse como un beneficio al empleador ya que entregaría un mayor nivel de certidumbre respecto de la duración del período de ausencia de la mujer que el que hoy existe. Por otro lado, un posnatal más largo significa una menor carga en costos de Sala Cuna para los empleadores que tienen en su planta a más de veinte mujeres. 
Además se considera importante plantear el acceso universal a Sala Cuna para los niños/as, ya que no todas las madres trabajan en empresas con más de veinte mujeres, por lo que este grupo de madres trabajadoras no cuenta con un recinto de atención de su bebé mientras trabajan, dejando esta labor a la cobertura de JUNJI e INTEGRA quienes no alcanzan a cubrir todas las demandas de matrícula y priorizan a las familias según la ficha de protección social. Por lo tanto, este grupo de madres al ser trabajadoras y tener "mejores" ingresos, se quedan sin cupo en las Salas Cuna gratuitas y no les alcanza para pagar una Sala particular. Entonces son las madres de la clase media las que presentan mayores inconvenientes para conciliar su maternidad con el trabajo.

Se transforma en una necesidad contar con mayor cobertura de Sala Cuna visualizando como país la necesidad de inversión en una Educación Parvularia de calidad. Es esta inversión en los niños/as lo que genera externalidades en el sentido de que gran parte del beneficio de la inversión afecta a la sociedad como un todo. Las inversiones en los niños generan externalidades positivas, es decir, cohesión social, confianza y reciprocidad al facilitar el trabajo femenino fuera del hogar y al mismo tiempo ayudar a conciliar trabajo y familia mediante la protección y promoción del bienestar de los niños/as mientras sus padres trabajan.

Este nivel educativo es uno de los más importantes para las investigaciones de las neurociencias y neurobiología, donde todo proceso de aprendizaje va acompañado de un cambio a nivel cerebral. Los bebés nacen con células cerebrales completas, pero las conexiones que se necesitan para cumplir el aprendizaje ocurren después del nacimiento teniendo una mayor plasticidad en los primeros tres años de vida y a su vez también durante estos años es el periodo crítico de la poda sináptica. Es por esto la importancia de una correcta estimulación de ambientes enriquecidos y la importancia de la Educación Parvularia y de las Salas Cuna no sólo como respuestas sociales asociadas al trabajo materno, sino como la real importancia de este nivel educativo, otorgando calidad por medio de experiencias educativas significativas y un clima organizado y acogedor de confianza y amor; realizando un cambio cultural en los adultos acerca las reales posibilidades del niño y niña. 


\section{Referencias bibliográficas}

Casas, L. y Valenzuela, E. (2012) Protección a la maternidad: una historia de tensiones entre los derechos de infancia y los derechos de las trabajadoras, en Revista de Derecho, 15, 1, 77-101.

Cortes, C.; Navarrete, E. y Troncoso, M. (2009). Construyendo experiencias desde la temprana infancia: Una Perspectiva Educacional Considerando la Neurociencia Santiago, Chile.

Mercedes, M.; Díaz, M. y Vásquez, J. (2016) ¿Demanda insuficiente o insatisfecha?: el caso de un programa municipal de provisión de guarderías en Chile / María Mercedes Mateo Díaz, Javiera Vásquez. Santiago, Chile.

Olhaberry, M. (2011) Calidad de la interacción madre-hijo(a), asistencia a Sala Cuna y la importancia de la edad de ingreso: estudio comparativo en familias monoparentales chilenas, en Revista Argentina de Clínica Psicológica, 161-173. 